\title{
Detonation Stabilization in Supersonic Flow: Effects of Suction Boundaries
}

\author{
Xiaodong Cai* \\ National University of Defense Technology, 410073 Changsha, People's Republic of China \\ Ralf Deiterding $\ddagger$ \\ University of Southampton, Southampton, England SO17 1BJ, United Kingdom \\ and \\ Jianhan Sun, \pm Mingbo Liang, $\underline{\S}$ and Dezun Dong $\mathbb{I}$ \\ National University of Defense Technology, 410073 Changsha, People's Republic of China
}

https://doi.org/10.2514/1.J058625

\begin{abstract}
In the present work, detonation stabilization in the supersonic flow is numerically investigated in the straight channel with suction boundaries. The two-dimensional reactive Navier-Stokes equations, together with a one-step reaction model, are solved using a second-order-accurate finite volume method solver based on the Structured Adaptive Mesh Refinement (SAMR) framework. The results show that, compared with one jet initiation, detonation initiation can be achieved in a shorter distance using two hot jets subject to the equal total width of the jets. When the suction slots are turned on, the overdriven detonation undergoes a gradual attenuation along with the weakening of the transverse waves, hence leading to dynamic detonation stabilization in the supersonic flow. When the suction slots are closer to the detonation front, the forward propagation of the detonation in the supersonic flow can be more effectively prohibited, implying that the suction slots should be distributed as close as possible to the detonation front in order to realize the maximal suction effect using the minimal suction slots.
\end{abstract}

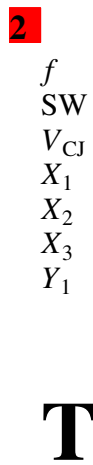

$\quad$ Nomenclature
$=$ overdrive degree
$=$ width of the suction slot, $\mathrm{cm}$
$=\quad$ Chapman-Jouguet velocity, $\mathrm{m} / \mathrm{s}$
$=\quad$ length of the straight channel, $\mathrm{cm}$
$=\quad$ distance from the hot jet edge to the outflow boundary, $\mathrm{cm}$
$=$ width of the hot jet, $\mathrm{cm}$
$=$ height of the channel, $\mathrm{cm}$

\section{Introduction}

$\mathbf{T}$ HANKS to the superior performance at high Mach numbers $(M a \geq 5.0)$, scramjets have become one of the first choices for hypersonic air-breathing propulsion systems. Because of the inherent theoretical advantage over deflagrative combustion adopted in scramjet combustors $[1,2]$, it is believed that the performance of the scramjet might be improved significantly if continuous detonationdriven combustion is realized effectively in scramjet combustors, that is, the detonation-driven scramjet (DDS) [3].

$\mathrm{Lu}$ and Braun [4] have pointed out that it is an important problem for practical detonation-driven engines to be able to sustain a detonation wave for a long duration and control it in some fashion when bringing the concept to reality. To put the DDS into practice, the first and foremost problem to be solved is the question of how to completely realize or at least dynamically control detonation stabilization

Received 15 May 2019; revision received 5 October 2019; accepted for publication 30 October 2019; published online XX epubMonth XXXX. Copyright $\odot 2019$ by the American Institute of Aeronautics and Astronautics, Inc. All rights reserved. All requests for copying and permission to reprint should be submitted to CCC at www.copyright.com; employ the eISSN 1533 $385 \mathrm{X}$ to initiate your request. See also AIAA Rights and Permissions www. aiaa.org/randp.

*Ph.D., College of Computer and Science and Technology on Scramjet Laboratory, Hunan; cai-chonger@ hotmail.com.

${ }^{\dagger}$ Associate Professor, Aerodynamics and Flight Mechanics Research Group, Highfield Campus; r.deiterding @ soton.ac.uk.

Frofessor, Science and Technology on Scramjet Laboratory, Hunan; jhleon@vip.sina.com (Corresponding Author).

${ }^{\S}$ Professor, Science and Technology on Scramjet Laboratory, Hunan; xd.cai .aero@outlook.com.

"Professor, College of Computer, Hunan; xdcaiaero@sina.com (Corresponding Author). in supersonic flows. However, detonation is a supersonic shock wave coupled with reaction zone that propagates normally at an average Chapman-Jouguet (CJ) velocity. It is therefore a great challenge to stabilize a detonation propagating at $\mathrm{CJ}$ state in supersonic combustors with limited length, if the incoming velocity is not close to the corresponding $\mathrm{CJ}$ velocity. When the incoming velocity is significantly larger or lower than the CJ velocity, the detonation will propagate forward or backward continuously, and cannot be effectively stabilized in the combustor.

Based on the idea of the DDS, in order to address detonation stabilization in supersonic flows the first approach is to use cavities, usually used as flame holders in supersonic combustors [5-7], which have been adopted for dynamic detonation stabilization in supersonic flows $[8,9]$. It is reported that the cavity can accelerate detonation propagation in supersonic combustible mixtures, thus finally resulting in overdriven detonation due to the enhancement of pressure oscillations caused by subsonic combustion in the cavity [8], and especially owing to the detonation wave interaction with the cavity, a periodical process that consists of forward propagation, detonation attenuation, and detonation self-sustainment is generated to avoid detonation failure [9]. Overall, it is suggested that the use of the cavity can contribute to the realization of detonation stabilization especially when the incoming velocity is larger than the CJ velocity. The second promising approach for sustained detonation stabilization with hot jet initiation is the use of expanding channels, which are usually adopted in actual propulsion combustors for maximizing thrust [10]. It is found that detonation can be maintained almost in the same position after the shutdown of the hot jet due to both the Prandtl-Meyer expansion and the rapid heat release of unburned jets through the turbulent mixing and diffusion [10]. The use of the expanding wall can help to realize detonation stabilization when the incoming velocity is lower than the $\mathrm{CJ}$ velocity especially for a relatively small expansion angle. Nevertheless, there are still some limitations that restrict the use of both the cavity and expanding wall into the actual control applications of detonation stabilization in supersonic flows. For instance, the cavity size plays an important role in detonation propagation, which has a significant influence on detonation stabilization [8]. However, it is difficult to dynamically change the cavity size for detonation stabilization control in actual experiments. For the expanding wall, detonation stabilization is not likely to be achieved in supersonic flows when the expansion angle is relatively 
large; furthermore, dynamically changing the expansion angle is a challenge in actual applications.

In addition to the cavity and expanding wall, an alternative method is the use of suction boundaries. The wall suction is among the first techniques applied to control the structure of the boundary layer. Most suction investigations concentrate on pure flow subject to active control by applying homogeneous suction boundaries [11-16]. Only a few studies are carried out concerning detonation combustion. Detonation experiments have been carried out in tubes with porous walls to investigate the dependence of detonability limits on the physical processes controlling the reaction rate in the detonation structure itself $[\underline{17,18]}$. When the detonation propagates through the porous walls, the permeability of the walls due to the suction effect can lead to mass leakage. The porous walls weaken the transverse waves, which further attenuate upon the wave reflection. The attenuation of transverse waves can largely result in the detonation failure for most detonation investigations in porous tubes, resulting from the suction effects of porous walls. It is reported that the porous walls act as an ideal loss boundary that simultaneously renders the wave one-dimensional by suppressing the multidimensional transverse wave structure, which can introduce the lateral mass divergence mechanism of failure [17]. Furthermore, the dependence of detonation propagation velocity on the diameter of porous tubes is investigated experimentally for two different mixtures with significantly different reaction zone structures, and the quenching of the detonation below a certain critical value of the tube diameter is illustrated [18]. Recently, a series of experiments have been conducted to investigate the failure mechanism of detonations in a porous tube [19]. It is reported that the successful transmission heavily relies on the frontal instability to generate local explosion centers for unstable turbulent detonations, whereas the failure is observed by the excessive global curvature for stable laminar detonations. The propagation of gaseous detonations in a channel with porous walls is investigated experimentally and numerically to determine the role of diffusive turbulent mixing and transverse waves in controlling the detonation limits in channels with porous walls [20]. It is found that due to the mass divergence into the damping section, resulting from the suction effect as the detonation propagates though the porous wall, the frontal wave curvature increases, whereas the number of transverse waves decreases in the porous section, which is caused by detonation attenuation.

All of the suction investigations concerning detonation combustion are carried out in quiescent reactive mixtures. To clarify the effects of suction boundaries on detonation propagation in supersonic flows, especially on the dynamic control of detonation stabilization, in the present work the robust second-order-accurate monotone upstream-centered schemes for conservation laws-total variation diminishing (MUSCL-TVD) scheme is applied based on an efficient structured adaptive mesh refinement (SAMR) framework [21,22]. Viscous detonation simulations with suction boundaries in supersonic flows are performed solving the reactive Navier-Stokes (NS) equations combined with a simple one-step reaction model. This work is part of an ongoing research program, aiming at providing information to help improve the overall understanding of dynamic stabilization of detonation in supersonic flows.

This paper is organized as follows: Sec. II introduces the computational model, including governing equations, numerical methods, and computational setup. Results and discussions are presented in Sec. III, where the initiation using two hot jets, dynamic detonation stabilization with suction boundaries, and the effects of suction positions are further investigated. Finally, Sec. IV concludes the paper.

\section{Computational Model}

\section{A. Governing Equations}

The present work uses the NS equations with the one-step chemistry model as governing equations [23]. Currently, it is very expensive to conduct high-resolution multidimensional detonation simulations solving the NS equations with detailed chemistry. The Arrhenius law that relates chemical reaction rates to temperature variation is widely used as the simplest mode for detonation simulations [24-26]. Here the reaction model is selected and fitted to the physical parameters of a
Table 1 Thermodynamic parameters of the mixture

\begin{tabular}{lcc}
\hline \hline Parameter & Value & Unit \\
\hline$T_{\infty}$ & 300 & $\mathrm{~K}$ \\
$p_{\infty}$ & 6.67 & $\mathrm{kPa}$ \\
$\rho_{\infty}$ & 0.077552 & $\mathrm{~kg} / \mathrm{m}^{3}$ \\
$\gamma$ & 1.29499 & \\
$W$ & 0.029 & $\mathrm{~kg} / \mathrm{mol}$ \\
$q$ & 54,000 & $\mathrm{~J} / \mathrm{mol}$ \\
$E_{a}$ & 30,000 & $\mathrm{~J} / \mathrm{mol}$ \\
$A$ & $6 \times 10^{5}$ & $\mathrm{~s}^{-1}$ \\
\hline \hline
\end{tabular}

$\mathrm{H}_{2} / \mathrm{O}_{2}$ detonation initially at $T=300 \mathrm{~K}$ and $P=6.67 \mathrm{kPa}$, to define a marginally stable detonation [27].

For the two species, the calorically perfect model

$$
\gamma=\gamma_{1}=\gamma_{2}, \quad p=\rho R T, \quad R=R_{1}=R_{2}
$$

is used. The mass fraction production rates are given as follows:

$$
w_{1}=-w_{2}=\rho Y_{1} A \exp \left(-\frac{E_{a}}{R T}\right)
$$

The thermodynamic parameters are shown in Table 1 and yield a CJ velocity of $V_{\mathrm{CJ}}=1587.84 \mathrm{~m} / \mathrm{s}$.

At the end of the Zel'dovich-von Neumann-Döring (ZND) reaction zone, the temperature is about $2500 \mathrm{~K}$ and the pressure is approximately $101.325 \mathrm{kPa}$, which gives the following transport parameters for the one-step model: $T_{\text {ref }}=2500 \mathrm{~K}, \mu_{\text {ref }}=1.07 \times 10^{-4} \mathrm{~Pa} \cdot \mathrm{s}$, $k_{\text {ref }}=0.148 \mathrm{~W} /(\mathrm{m} \cdot \mathrm{K}), D_{1 \mathrm{ref}}=5.5 \times 10^{-4} \mathrm{~m}^{2} / \mathrm{s}, D_{2 \mathrm{ref}}=6.4 \times$ $10^{-4} \mathrm{~m}^{2} / \mathrm{s}$. By matching the general trends and values at the end of the ZND reaction zone between the one-step and detailed reaction model, the viscosity and conductivity are given by the Sutherland model, whereas the mass diffusion is given by a simple expression, which includes the inverse dependence on pressure.

$$
\begin{array}{cc}
\frac{u}{u_{\text {ref }}}=\left(\frac{T}{T_{\text {ref }}}\right)^{5 / 2}, & \frac{k}{k_{\text {ref }}}=\left(\frac{T}{T_{\text {ref }}}\right)^{5 / 2} \\
\frac{D_{1}}{D_{\text {1ref }}}=\left(\frac{T}{T_{\text {ref }}}\right)^{5 / 2} \frac{p_{\text {atm }}}{p}, & \frac{D_{2}}{D_{\text {2ref }}}=\left(\frac{T}{T_{\text {ref }}}\right)^{5 / 2} \frac{p_{\text {atm }}}{p}
\end{array}
$$

\section{B. Numerical Methods}

Dimensional splitting is employed for the two-dimensional simulations. For the reactive simulations, a time-splitting method is used due to the large difference in time scales between the fluid dynamics and reactive source term.

The second-order-accurate MUSCL-TVD finite volume method is adopted for discretization of the convective part of the NS equations, whereas the second-order-accurate centered difference (CD) scheme is used for the diffusive term. This has been effectively validated for multidimensional detonations [27]. Unlike shock-capturing methods, no numerical viscosity is introduced for schemes based on centered stencils, thus being able to accurately describe the physical diffusion. As for the reactive source term, a semi-implicit, A-stable generalized Runge-Kutta method of fourth order (GRK4A) is used for the integration, avoiding a globally coupled implicit problem.

Additional restrictions are imposed on the time integration by the $\mathrm{CD}$ scheme. It is relatively easy to use explicit multistage time integration methods for the implementation with SAMR. Because of the stability properties of the explicit integration schemes, the preferred practical methods with the ability of inexpensive time adaption in SAMR are Runge-Kutta methods. Here, the optimal second-orderaccurate strong stability preserving Runge-Kutta scheme is used with a CFL parameter of 0.98 . 


\section{Computational Setup}

The computations are carried out in two-dimensional straight channels, as depicted in Fig. 1. Reflecting boundaries with slip wall conditions are used on the upper and lower walls. In the viscid case, a turbulent boundary layer would develop along the inflow channel wall and its thickness will strongly depend on the length of the channel. This can accurately be described only by either simulating the entire combustion chamber or employing an analytic boundarylayer profile as a nontrivial inflow condition. To eliminate this complexity and to be able to focus the present study just on the effects of suction boundaries on detonation propagation and the subsequent stabilization control in the supersonic flow, the viscid model with slip boundary conditions is opted for not having to deal with boundary layers produced otherwise in the inflow section. An inflow condition is set up to model a hot jet, which is symmetrically located on both the upper and the lower walls. Throughout the channel the supersonic reactive flow enters from right to left at the corresponding CJ velocity. The right boundary adopts the inflow condition and an ideal outflow condition is imposed on the left boundary.

The suction holes are uniformly distributed symmetrically on both the lower and the upper walls. Suction can be applied by some mechanical means; however, passive suction is usually preferred because of its simplicity. Passive suction does not require any energy expenditure, which can be achieved by using the pressure differential between locations. In the present study, passive suction is adopted by using the suction slots. Six suction slots $\left(S_{1}, S_{2}, S_{3}, S_{4}, S_{5}, S_{6}\right)$ with the same width are uniformly distributed symmetrically on both the lower and the upper walls, where a constant pressure is prescribed at the holes. The velocity and momentum are still allowed to reflect as usual, whereas the remaining state variables are implemented by constant-value extrapolation of the interior data into the ghost cells. The given pressure of the slots is relatively low with respect to that in the supersonic flow. Therefore, the fluid in the channel could be removed through the slots without additional power requirements.

The inflow parameters of the hot jet are set to the values of the CJ state of a $\mathrm{H}_{2} / \mathrm{O}_{2}$ detonation under the condition of pressure $6.67 \mathrm{kPa}$ and temperature $300 \mathrm{~K}$. The injection velocity is specified as the sonic speed to make it a choked hot jet. The details of the hot jet are shown in Table $\underline{2}$.

\section{Convergence Analysis}

As shown in Fig. 1, the length and height of the channel are $X_{1}=$ $75 \mathrm{~mm}$ and $Y_{1}=25 \mathrm{~mm}$, respectively. The distance between the hot jet and the head wall is $X_{2}=25 \mathrm{~mm}$, and the width of the hot jet is $X_{3}=2 \mathrm{~mm}$. The slots with the same width of SW $=2 \mathrm{~mm}$ and the initial pressure $5 \mathrm{kPa}$ are uniformly placed with the interval of $5 \mathrm{~mm}$ symmetrically on both the lower and the upper walls. The first slot $\left(S_{1}\right)$ is $2.9 \mathrm{~mm}$ away from the hot jet. The initial mesh has $600 \times 200$ cells, which gives an initial grid resolution of $1.25 \times 10^{-4} \mathrm{~m}$ in both directions.

Three different cases of SAMR implementation are carried out to investigate the effect of grid resolution. The detailed information of the three cases is shown in Table $\underline{3}$.

After the injection of the two impinging hot jets into the supersonic flow, two symmetrical bow shocks are first induced and eventually a Mach stem is generated after the collision of the bow shock waves. Figure 2 shows three different distributions of mesh refinement for the typical structure of the Mach stem induced by the injection of the two symmetrical jets. The overall pattern associated with the triple points resulting from the Mach stem, incident shocks, and slip lines

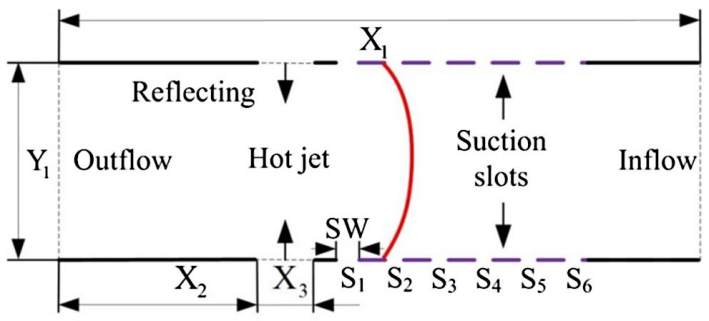

Fig. 1 Schematic of the calculation model.
Table 2 Equilibrium CJ state of the hot jet

\begin{tabular}{lcc}
\hline \hline Parameter & Value & Unit \\
\hline Pressure & 86,376 & $\mathrm{~Pa}$ \\
Temperature & $1,943.8$ & $\mathrm{~K}$ \\
Density & 0.155 & $\mathrm{~kg} / \mathrm{m}^{3}$ \\
Velocity & 850 & $\mathrm{~m} / \mathrm{s}$ \\
Total energy & 349,280 & $\mathrm{~J} / \mathrm{mol}$ \\
$Y_{1}$ (reactant mass fraction) & 0.0088 & \\
$Y_{2}$ (product mass fraction) & 0.9912 & \\
\hline \hline
\end{tabular}

Table 3 Mesh refinement parameters

\begin{tabular}{lccc}
\hline \hline & Case 1 & Case 2 & Case 3 \\
\hline \multirow{3}{*}{ Refinement } & 3 levels & 4 levels & 5 levels \\
parameters & $(2,4)$ & $(2,2,4)$ & $(2,2,2,4)$ \\
& $\Delta_{\min }=$ & $\Delta_{\min }=$ & $\Delta_{\min }=$ \\
& $1.56 \times 10^{-5} \mathrm{~m}$ & $7.8 \times 10^{-6} \mathrm{~m}$ & $3.9 \times 10^{-6} \mathrm{~m}$ \\
\hline \hline
\end{tabular}

emitting from the triple points is qualitatively similar in the three cases with different grid resolutions. Note that these characteristic structures are all captured by the highest refinement levels, indicating that the refinement strategy established in the simulations can satisfy the requirements. As a compromise between computational cost and resolved resolution, the four-level mesh refinement of case 2 is adopted in the following simulations.

The highest grid resolution in case 2 of $\Delta_{\min }=7.8 \times 10^{-6} \mathrm{~m}$ corresponds to approximate $275 \mathrm{Pts} / \mathrm{Lhr}$ (points per half reaction length). This has been effectively verified in the recent work [10], which shows that at least about 10 cells can be placed within the viscous scale, hence indicating that these diffusive scales can be fully resolved in the present simulations except for shock waves whose thicknesses are only several mean free path lengths.

\section{A. Initiation Using Two Hot Jets}

\section{Results and Analysis}

Detonation initiation using one hot jet in the supersonic flow has been fully investigated in the previous work [10]. Different from the sole jet initiation, in the present work two symmetrical hot jets are used to avoid the potential unsymmetrical disturbance on the suction boundaries. To obtain an overall understanding of this issue, detonation initiation in the supersonic flow using two jets is illustrated in Fig. 3.

After the injection of the two hot jets into the supersonic flow, two symmetrical bow shock waves are generated and further collide with each other in the middle of the channel, as shown in Fig. 3a. Subsequently, as the strength of the reflected shock waves increases, Mach reflections are produced on both the upper and the lower walls. As shown in Fig. 3b, the two Mach reflections begin to interact with each other, resulting in the generation of interacting expansion and shock waves inside the hydrodynamic channel formed by the two slip lines emitting from the triple points. The Mach reflections propagate forward further, and eventually the two triple points collide to generate a new Mach stem, as shown in Fig. 3c.

Figure 4 visualizes the detailed structure of the local Mach stem using the contour of the product mass fraction. As shown in Fig. 4a, it is observed between the two primary triple points that the Mach stem is coupled tightly with the reaction front, indicating the formation of a Mach detonation (Mach-stem-induced detonation) [28]. In addition to the primary triple points, the secondary ones are also resolved associated with the expansion waves resulting from the shock reflections on the slip lines emitting from the primary triple points, suggesting adequate resolution in the present work. Compared with that in Fig. 4a, the Mach detonation in Fig. 4b has propagated forward. The slip lines developed to highly unstable shear layers can be considered as free boundaries, hence forming a convergent channel behind the Mach detonation. It is reported that highly unstable shear layers are able to enhance the diffusion and mixing effects [29], which can significantly contribute to the consumption and subsequent heat 


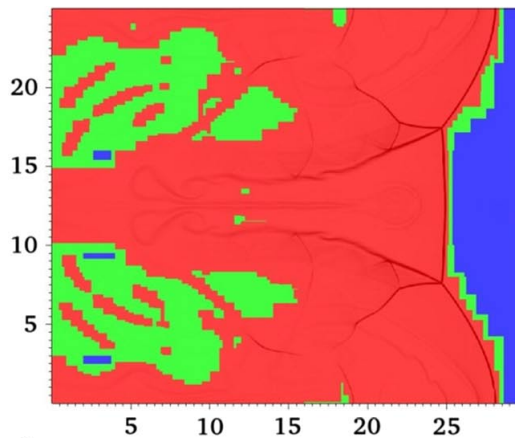

a) b)

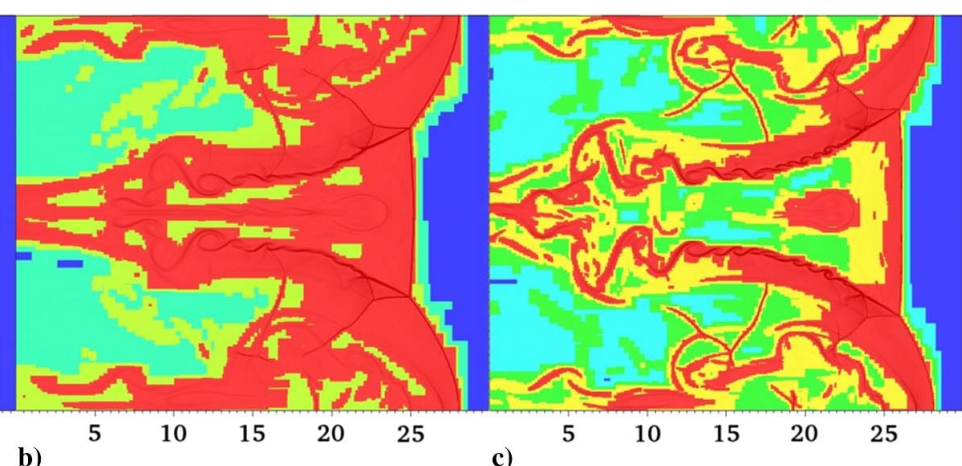

Fig. 2 Three refinement cases: a) case 1, b) case 2, c) case 3.

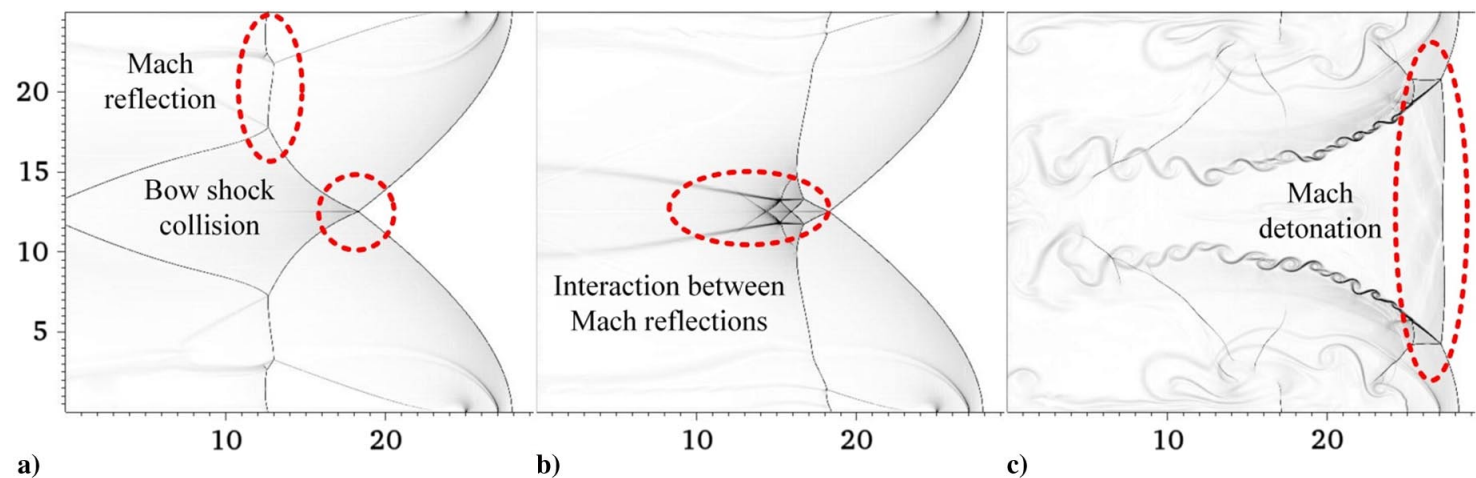

a)

Fig. 3 Detonation initiation using two hot jets: a) $t=100 \mu \mathrm{s}$, b) $t=150 \mu \mathrm{s}$, c) $t=200 \mu \mathrm{s}$.

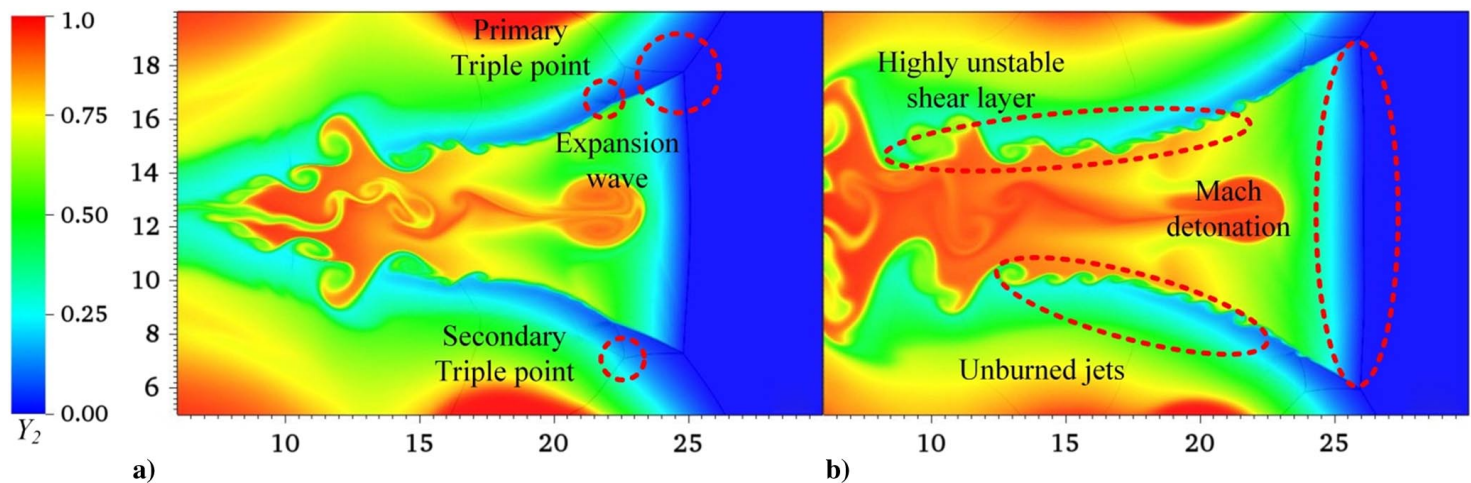

Fig. 4 The detailed structure of Mach detonation: a) $t=185 \mu \mathrm{s}$, b) $t=190 \mu \mathrm{s}$.

release of the unburned jets behind the Mach detonation. This can facilitate the forward propagation of the Mach detonation, resulting in an overdriven detonation in the supersonic flow. It can be calculated that the relative velocity is approximately $v=200 \mathrm{~m} / \mathrm{s}$ with the corresponding overdrive degree of $f=1.268\left(f=\left[\left\{V_{\mathrm{CJ}}+v\right\} / V_{\mathrm{CJ}}\right]^{2}\right)$. The generation of the local Mach detonation finally can lead to the full detonation in the whole channel.

A similar setup is conducted for comparison to investigate the initiation characteristic directly using two hot jets. The new setup uses only one hot jet on the lower wall with the width $X_{3}=4 \mathrm{~mm}$, ensuring an identical total width of the hot jets, whereas the other conditions remain unaltered.

As shown in Fig. 5, it is found that detonation initiation is successfully realized at approximately the same time of $t=165 \mu \mathrm{s}$, and after the successful initiation the front trajectories are almost parallel using the two initiation methods, indicating the same relative velocity of forward propagating. The difference is that, when using two jets, detonation initiation can be achieved within a distance $8 \mathrm{~mm}$ shorter than that using one jet initiation, which is useful for actual applications.

\section{B. Stationary Propagation with Suction Boundaries}

Figure $\underline{6}$ shows the propagation of the detonation after the triggering of the suction slots. After the initiation, the overdriven detonation

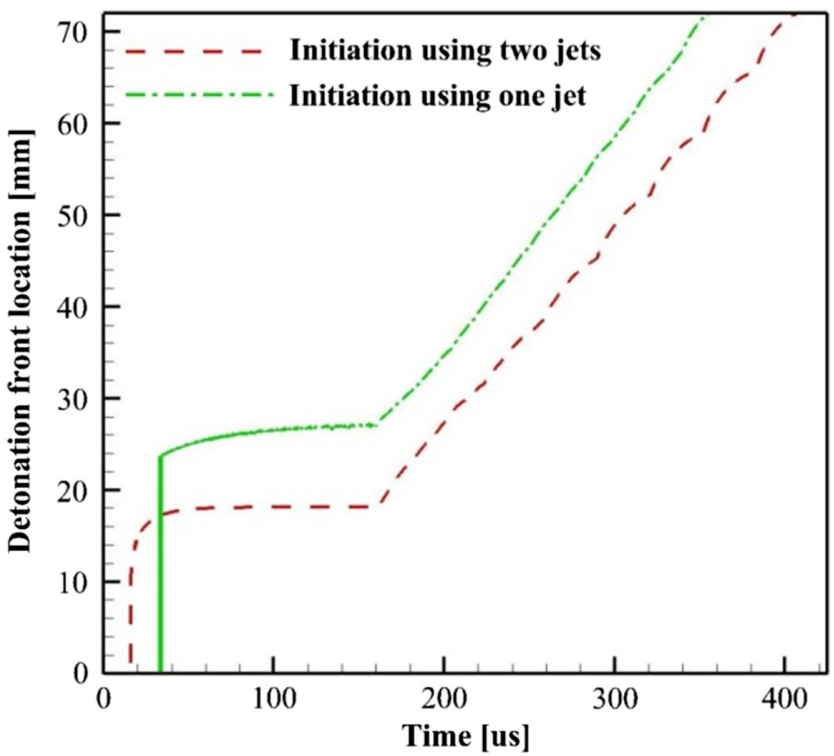

Fig. 5 The trajectories of the detonation fronts using both the two initiation methods. 

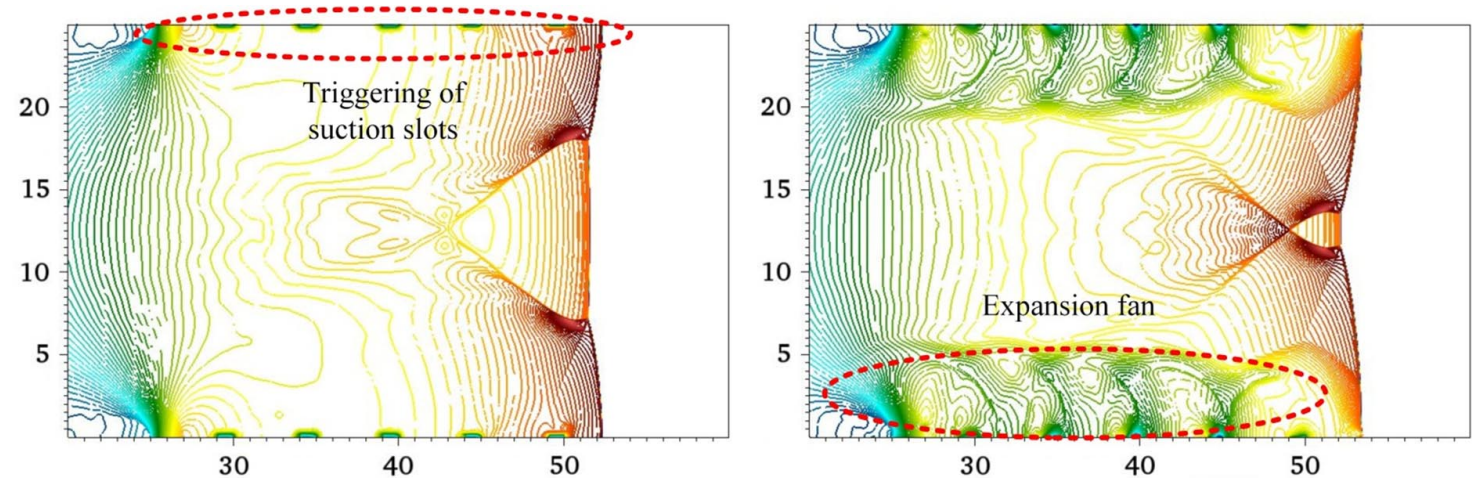

a)

b)
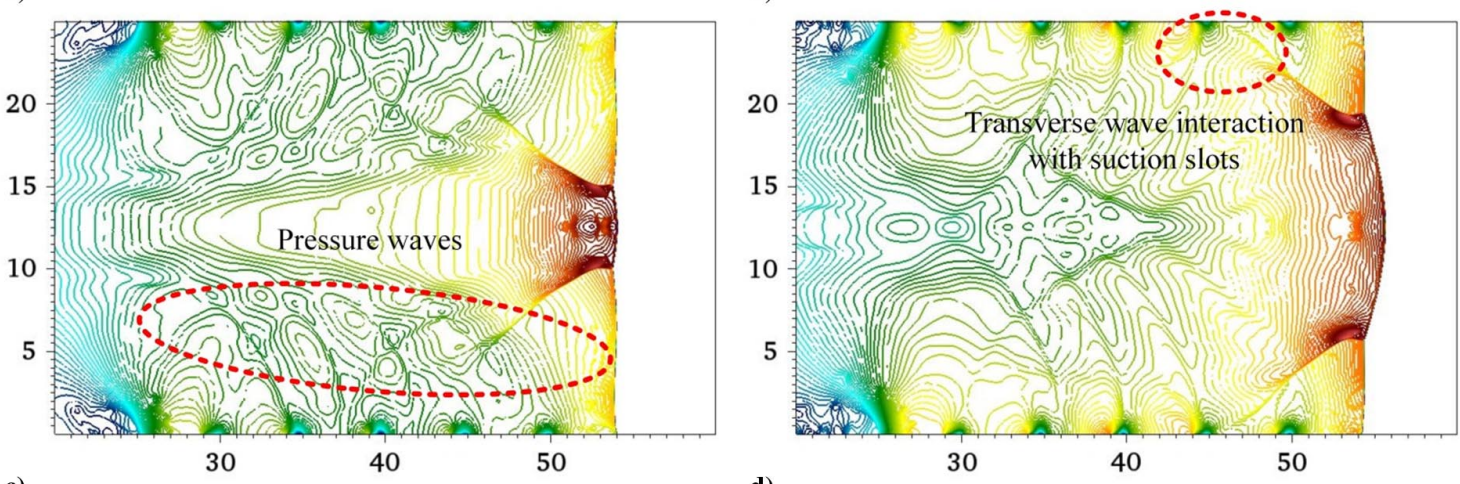

c)

d)

Fig. 6 Pressure isolines depicting detonation propagation immediately after triggering of the suction slots: a) $t=315 \mu \mathrm{s}$, b) $t=320 \mu \mathrm{s}$, c) $t=325 \mu \mathrm{s}$, d) $t=330 \mu \mathrm{s}$.

continues the forward propagation. At $t=315 \mu \mathrm{s}$, the suction boundary is triggered to open the six suction slots $\left(S_{1}, S_{2}, S_{3}, S_{4}, S_{5}, S_{6}\right)$, as shown in Fig. 6a. At this time, it is ensured that the six suction slots are all located behind the detonation front. After the triggering, it is observed that the expansion waves are immediately generated on the upper and lower walls near the slots as shown in Fig. 6b, which further will result in numerous pressure oscillations associated with the formation of pressure waves behind the detonation front, as shown in Fig. 6c. In addition, in Fig. 6d, it can be observed that the transverse waves are interacting with the suction slots. Because of the mass divergence resulting from the suction slots that generate the expansion waves, the transverse waves might be further weakened.

Figure 7 shows the detailed structures of detonation propagation with open suction slots. Because of the symmetry of the flowfield, only the upper half part is illustrated.

In Fig. 7a, it is observed that a double Mach reflection with a strong transverse wave is generated. Subsequently, this double Mach refection turns to propagate upward after the collision with another triple

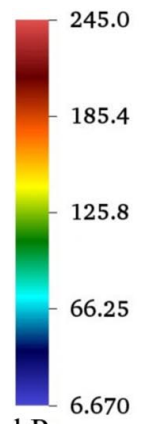

$\mathrm{kPa}$

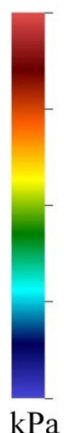

66.25

6.670

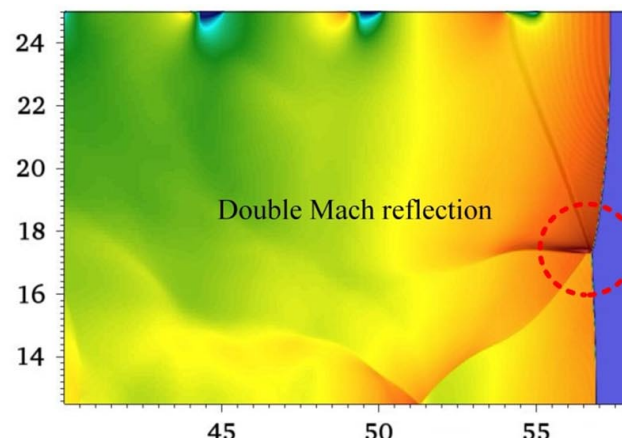

a)

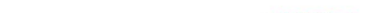

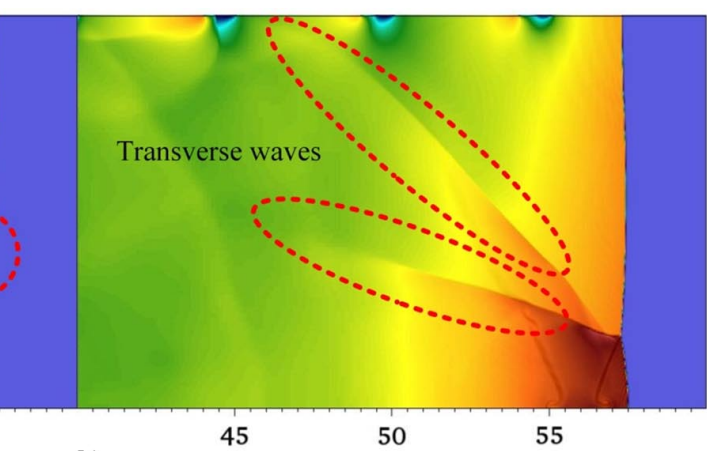

b)
Transverse wave interaction with suction slots

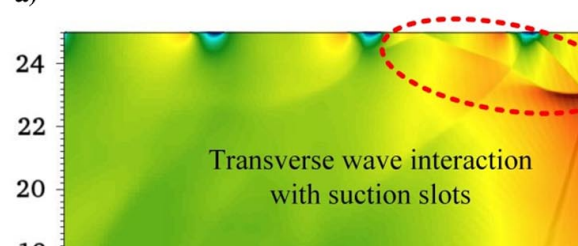

c)

Fig. 7 Detailed detonation structures with suction boundaries: a) $t=350 \mu \mathrm{s}$, b) $t=360 \mu \mathrm{s}$, c) $t=370 \mu \mathrm{s}$, d) $t=380 \mu \mathrm{s}$. 

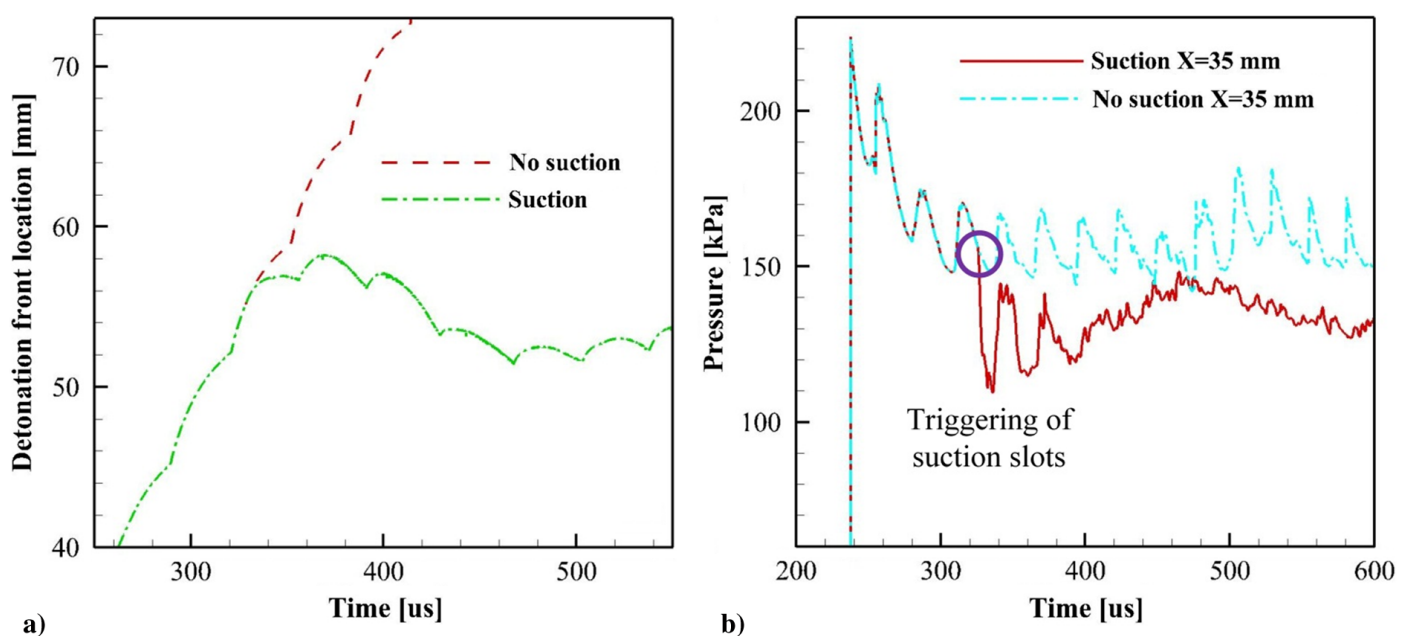

Fig. 8 Comparison between suction and no suction boundaries: a) trajectories of the detonation front both with and without the suction boundary; b) pressures behind the detonation front both with and without the suction boundary at $X=35 \mathrm{~mm}, Y=12.5 \mathrm{~mm}$.

point in the middle. As shown in Fig. 7b, two transverse waves are clearly distinguished from the double $\overline{M a c h}$ reflection, which eventually leads to reflections on the upper wall. It is observed in Fig. 7c that the lower transverse wave just swept from the slot $S_{5}$, whereas the upper transverse wave is interacting with the slot $S_{6}$. In addition to the transverse wave interactions with suction slots, the slip lines can also interact with the slots, as shown in Fig. 7d. As a consequence of the suction, the transverse waves weaken when interacting with the slots. Compared with that in Fig. 7a, the maximal pressure in Fig. $7 \mathrm{~b}$ behind the transverse wave decreases about $20 \%$, resulting in a reduction $16 \%$ of the transverse wave strength. This also has a significant influence on the Mach reflection pattern. It is found that the strong double Mach reflection structure in Fig. 7a is gradually replaced by weaker ones and even transitional Mach reflection in Fig. 7d.

Figure $8 \mathrm{a}$ illustrates the trajectories of the detonation fronts both with and without the suction boundaries. It is found that when there is no suction boundary the detonation wave continues its forward propagation with an overdrive degree of $f=1.29$ with an oscillating period of $T=31.26 \mu \mathrm{s}$. However, when the suction slots are opened the detonation wave gradually changes from the forward propagation to dynamically stationary propagation with an oscillating period of $T=35.2 \mu \mathrm{s}$, which is $12.6 \%$ larger than that with no suction. Through this comparison, it is indicated that using the suction boundaries it is likely to realize the dynamic stabilization of detonation in the supersonic flow.

Figure $8 \mathrm{~b}$ shows the pressure variations at a fixed point in the middle of the channel behind the detonation front both with and without the suction. It is found that, when there is no suction boundary, the pressure behind the detonation front presents a periodic oscillation with a relative constant period of $\Delta T=7.68 \mu$ s and oscillating amplitude $\Delta p=17.4 \mathrm{kPa}$. The oscillating period results from the periodic movement of the two transverse waves behind the detonation front as shown in Fig. 6. This also can evaluate the detonation oscillation period as $T=4 \overline{\times} \Delta T=30.72 \mu \mathrm{s}$, which is very close to that in Fig. 8a. However, when the suction boundary is opened, the pressure first undergoes a sharp decrease and then turns to an irregular oscillation without an obvious period and oscillating amplitude. Overall, the pressure after the suction triggering is approximately $30 \mathrm{kPa}$ lower than that with no suction. The suction effect results in a decline of the pressure behind the detonation wave. The pressure oscillation behind the detonation is a representation of the transverse wave movement.

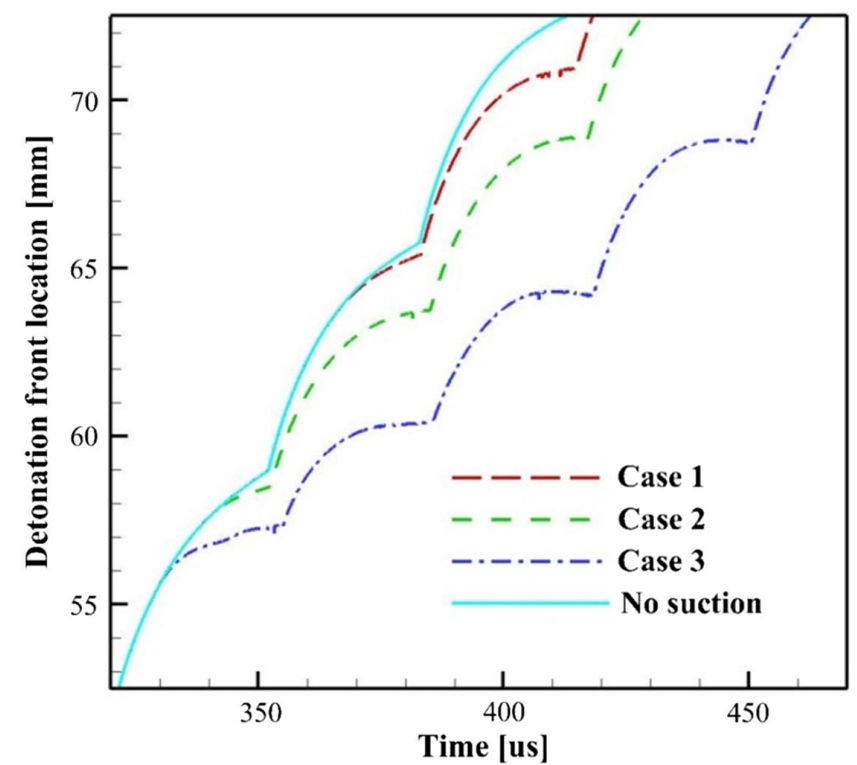

Fig. 9 Trajectories of the detonation fronts for different positions of suction slots.

This can further lead to the chemical reaction slowdown and the subsequent heat release. Detonation propagation requires the support of the energy released from the reaction behind the wave, yet it is not possible at this point for the chemical reaction to continuously support the propagation of an overdriven detonation wave. Therefore, during the propagation, the overdriven detonation undergoes an attenuation along with the weakening of the transverse waves, hence leading to dynamically stationary propagation of detonation in the supersonic flow associated with irregular pressure oscillations.

\section{Effect of Suction Position}

To provide a comprehensive understanding of the suction effects on detonation propagation in supersonic flows, a systematic study should be carried out. Except for the strength of the suction slots whose effects are very straightforward, the suction position is the

Table 4 Setups for the three cases

\begin{tabular}{ccccccc}
\hline \hline & $S_{1}$ & $S_{2}$ & $S_{3}$ & $S_{4}$ & $S_{5}$ & $S_{6}$ \\
\hline Case 1 & $\sqrt{ }$ & $\sqrt{ }$ & $\times$ & $\times$ & $\times$ & $\times$ \\
Case 2 & $\times$ & $\times$ & $\sqrt{ }$ & $\sqrt{ }$ & $\times$ & $\times$ \\
Case 3 & $\times$ & $\times$ & $\times$ & $\times$ & $\sqrt{ }$ & $\sqrt{ }$ \\
\hline \hline
\end{tabular}

$\sqrt{ }$, opening of the suction slot; $\times$, shutdown.

Table 5 Corresponding parameters for the four cases

\begin{tabular}{lccc}
\hline \hline & $T(\mu \mathrm{s})$ & $v(\mathrm{~m} / \mathrm{s})$ & $f$ \\
\hline Case 1 & 31.99 & 174.6 & 1.23 \\
Case 2 & 32.48 & 161.5 & 1.21 \\
Case 3 & 32.97 & 112.8 & 1.15 \\
No suction & 31.26 & 220 & 1.29 \\
\hline \hline
\end{tabular}



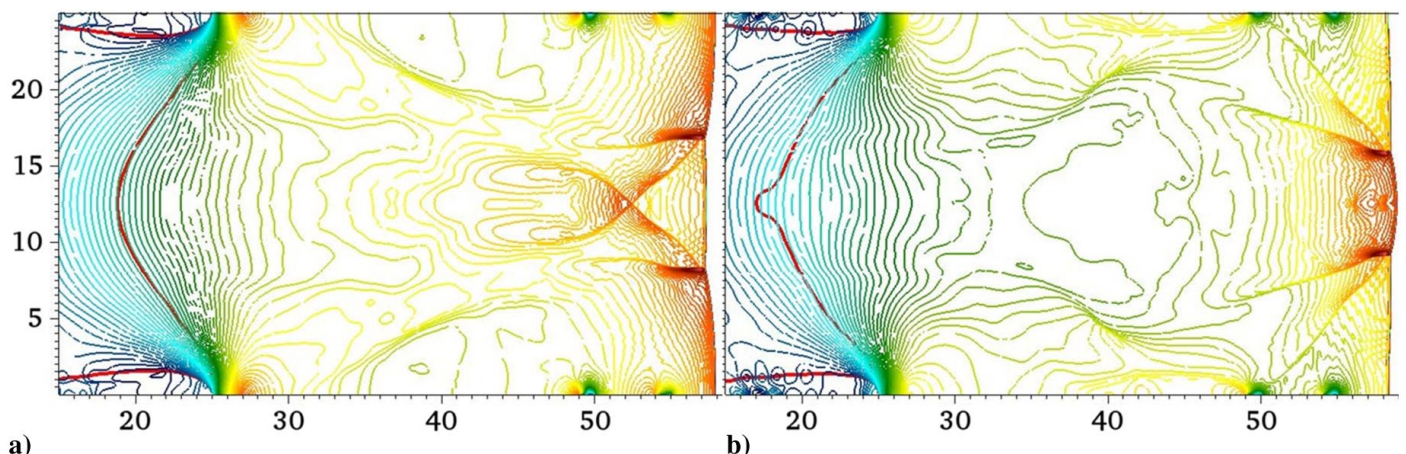

Fig. 10 Sonic lines for case 3: a) $t=350 \mu \mathrm{s}$, b) $t=360 \mu \mathrm{s}$.

most important for detonation propagation in supersonic flows. A proper suction position can realize the maximal suction effect using the least slots. Therefore, the following investigation concentrates on the distribution of the suction slots.

To investigate the effect of the suction position, three cases are carried out, which are listed in Table 4 . For case 1, only slots $S_{1}$ and $S_{2}$ are opened, whereas the other slots are all shut down; for case 2 , only slots $S_{3}$ and $S_{4}$ are opened, whereas the other slots are all shut down; for case 3 , only slots $S_{5}$ and $S_{6}$ are opened, whereas the other slots are all shut down.

Figure 9 shows the trajectories of the detonation fronts for the three cases. It is observed that all the trajectories undergo a downward deviation after the suction slots are opened. Subsequently, the slopes of the trajectories gradually become relatively stable associated with periodical oscillations. However, due to the different locations of the suction slots, the slopes of the three curves that indicate the relative propagation velocities are different.

The corresponding oscillating periods, relative velocities, and overdrive degrees for the four cases are listed in details in Table $\underline{5}$. It is shown that from case 1 to case 3 , the oscillating period presents an increasing trend, whereas the relative velocity and overdrive degree decrease slightly. Compared with the case of no suction, the period increases about $2.28,3.9$, and $5.47 \%$, which is also an indication of the weakening of the transverse waves, whereas the overdrive degree decreases about $4.65,6.2$, and $10.85 \%$, respectively. It is found that although the equal two suction slots are used in the three cases, different distribution positions lead to quite different suction effects. When the suction slots are closer to the detonation front, the forward propagation of the detonation in the supersonic flow can be more effectively prohibited, indicating the more obvious suction effects.

A sonic line is produced between the hot jet and the detonation front in supersonic flows [30,31]. Hence, a subsonic area exits between the sonic line and the detonation front. If the suction slots are distributed in the subsonic area, the suction effect can effectively play a significant role in the detonation front crossing through the subsonic area. Otherwise, the suction effect cannot effectively influence the detonation propagation in the supersonic region downstream in the subsonic area.

As indicated in Table 4, the suction effect becomes more significant when the suction slot is getting closer to the detonation front. As shown in Fig. 10, the sonic lines of case 3, where the suction effect is the most significant, are illustrated by the red lines. This implies that, when using the suction boundary for the control of detonation propagation in supersonic flows, the suction slots should be distributed as close as possible to the detonation front in order to realize the maximal effect using the minimal suction slots.

\section{Conclusions}

In the present work, detonation stabilization in the supersonic flow is investigated in the straight channel with suction boundaries. Two symmetrical hot jets are used for the initiation in the supersonic flow. The reactive NS equations are solved together with a one-step reaction model based on an SAMR framework. Under the given circumstance the main results are as follows:
1) After the reflections of bow shock waves induced from the injection of two symmetrical hot jets into the supersonic flow, a Mach stem is generated, which is actually a local Mach detonation indicating the successful initiation. Compared with one jet initiation, the initiation is successfully realized in a shorter distance subject to the equal total width of the jets when using two hot jets.

2) When triggering the suction slots, the overdriven detonation gradually undergoes an attenuation associated with the weakening of the transverse waves due to the suction effects. Eventually, dynamically stationary propagation of detonation is generated in the supersonic flow together with irregular pressure oscillations.

3) Different distribution positions of suction slots can lead to quite different suction effects. When the suction slots are closer to the detonation front, the forward propagation of detonation in the supersonic flow can be more effectively prohibited. This implies that, when using the suction boundary for the control of detonation propagation in supersonic flows, the suction slots should be distributed as close as possible to the detonation front in order to realize the maximal effect using the minimal number of suction slots.

\section{Acknowledgments}

This work was supported by National Natural Science Foundation of China (No. 11702323), Postdoctoral Innovative Talent Support Program (No. BX20180372), and National Foundation of Defense Technology (No. 3101032).

\section{References}

[1] Kailasanath, K., "Review of Propulsion Applications of Detonation Waves," AIAA Journal, Vol. 38, No. 9, 2000, pp. 1698-1708. https://doi.org/10.2514/2.1156

[2] Wolański, P., "Detonative Propulsion," Proceedings of the Combustion Institute, Vol. 34, No. 1, 2013, pp. 125-158. https://doi.org/10.1016/j.proci.2012.10.005

[3] Cai, X. D., Liang, J. H., Deiterding, R., Mahmoudi, Y., and Sun, M. B., "Experimental and Numerical Investigations on Propagating Modes of Detonations: Detonation Wave/Boundary Layer Interaction," Combustion and Flame, Vol. 190, April 2018, pp. 201-215. https://doi.org/10.1016/i.combustflame.2017.11.015

[4] Lu, F. K., and Braun, E. M., "Rotating Detonation Wave Propulsion: Experimental Challenges, Modeling, and Engine Concepts," Journal of Propulsion and Power, Vol. 30, No. 5, 2014, pp. 1125-1142. https://doi.org/10.2514/1.B34802

[5] Yeom, H. W., Seo, B. G., and Sung, H. G., "Numerical Analysis of a Scramjet Engine with Intake Sidewalls and Cavity Flameholder," AIAA Journal, Vol. 51, No. 7, 2013, pp. 1566-1575. https://doi.org/10.2514/1.J051677

[6] Colcord, B. J., Sirignano, W. A., and Liu, F., "Flameholding in Converging and Turning Channels over Cavities with Periodic Port Injection," AIAA Journal, Vol. 51, No. 7, 2013, pp. 1621-1630. https://doi.org/10.2514/1.J051890

[7] Kirik, J. W., Goyne, C. P., Peltier, S. J., Carter, C. D., and Hagenmaier, M. A., "Velocimetry Measurements of a Scramjet Cavity Flameholder with Inlet Distortion," Journal of Propulsion and Power, Vol. 30, No. 6, 2014, pp. 1568-1576. https://doi.org/10.2514/1.B35195

[8] Cai, X. D., Liang, J. H., Deiterding, R., and Lin, Z. Y., "Adaptive Simulations of Cavity-Based Detonation in Supersonic Hydrogen-Oxygen 
Mixture," International Journal of Hydrogen Energy, Vol. 41, No. 16, 2016, pp. 6917-6928.

https://doi.org/10.1016/j.ijhydene.2016.02.144

[9] Cai, X. D., Liang, J. H., Deiterding, R., Lin, Z. Y., and Sun, M. B., "Detonation Interaction with Cavity in Supersonic Combustible Mixture," AIAA Journal, Vol. 56, No. 5, 2018, pp. 2096-2102. https://doi.org/10.2514/1.J056392

[10] Cai, X. D., Deiterding, R., Liang, J. H., and Mahmoudi, Y., "Adaptive Simulations of Viscous Detonations Initiated by a Hot Jet Using a HighOrder Hybrid WENO-CD Scheme," Proceedings of the Combustion Institute, Vol. 36, No. 2, 2017, 2725-2733.

https://doi.org/10.1016/j.proci.2016.06.161

[11] Khapko, T., Schlatter, P., Duguet, Y., and Henningson, D. S., "Turbulence Collapse in a Suction Boundary Layer," Journal of Fluid Mechanics, Vol. 795, May 2016, pp. 356-379. https://doi.org/10.1017/jfm.2016.205

[12] Kreilos, T., Gibson, J. F., and Schneider, T. M., "Localized Travelling Waves in the Asymptotic Suction Boundary Layer," Journal of Fluid Mechanics, Vol. 795, May 2016, p. R3. https://doi.org/10.1017/ifm.2016.242

[13] Wahidi, R., and Bridges, D. H., "Effects of Distributed Suction on an Airfoil at Low Reynolds Number," AIAA Journal, Vol. 50, No. 3, 2012, pp. 523-539. https://doi.org/10.2514/1.J050913

[14] Arnold, B., Lutz, T., Krämer, E., and Rautmann, C., "Wind-Turbine Trailing-Edge Noise Reduction by Means of Boundary-Layer Suction," AIAA Journal, Vol. 56, No. 5, 2018, pp. 1843-1854. https://doi.org/10.2514/1.J056633

[15] Vigdorovich, I., "A Law of the Wall for Turbulent Boundary Layers with Suction: Stevenson's Formula Revisited," Physics of Fluids, Vol. 28, No. 8, 2016, Paper 085102. https://doi.org/10.1063/1.4960182

[16] Pluvinage, F., Kourta, A., and Bottaro, A., "Instabilities in the Asymptotic Suction Boundary Layer over a Permeable, Compliant Wall," Physics of Fluids, Vol. 27, June 2015, Paper 054104. https://doi.org/10.1063/1.4921422

[17] Radulescu, M. I., and Lee, J. H. S., "The Failure Mechanism of Gaseous Detonations: Experiments in Porous Wall Tubes," Combustion and Flame, Vol. 131, Nos. 1-2, 2002, pp. 29-46. https://doi.org/10.1016/S0010-2180(02)00390-5

[18] Radulescu, M. I., "The Propagation and Failure Mechanism of Gaseous Detonations: Experiments in Porous-Walled Tubes," Ph.D. Thesis, McGill Univ., Montreal, 2003.

[19] Mehrjoo, N., Gao, Y., Kiyanda, C. B., Ng, H. D., and Lee, J. H. S., "Effects of Porous Walled Tubes on Detonation Transmission into Unconfined Space," Proceedings of the Combustion Institute, Vol. 35, No. 2, 2015, pp. 1981-1987. https://doi.org/10.1016/j.proci.2014.06.031

[20] Mazaheri, K., Mahmoudi, Y., Sabzpooshani, M., and Radulescu, M. I., "Experimental and Numerical Investigation of Propagation Mechanism of Gaseous Detonations in Channels with Porous Walls," Combustion and Flame, Vol. 162, No. 6, 2015, pp. 2638-2659. https://doi.org/10.1016/j.combustflame.2015.03.015

[21] Deiterding, R., "Parallel Adaptive Simulation of Multi-Dimensional Detonation Structures," Ph.D. Dissertation, Brandenburg Univ. of Technology Cottbus, Cottbus, Germany, 2003.

[22] Cai, X. D., Liang, J. H., Lin, Z. Y., Deiterding, R., Qin, H., and Han, X., "Adaptive Mesh Refinement Based Numerical Simulation of Detonation Initiation in Supersonic Combustible Mixtures Using a Hot Jet," ASCE Journal of Aerospace Engineering, Vol. 28, No. 1, 2015, Paper 04014046.

https://doi.org/10.1061/(ASCE)AS.1943-5525.0000376

[23] Cai, X. D., Deiterding, R., Liang, J. H., Sun, M. B., and Mahmoudi, Y., "Diffusion and Mixing Effects in Hot Jet Initiation and Propagation of Hydrogen Detonations," Journal of Fluid Mechanics, Vol. 836, Feb. 2018, pp. 324-351 https://doi.org/10.1017/jfm.2017.770

[24] Bane, S. P. M., Ziegler, J. L., and Shepherd, J. E., "Development of OneStep Chemistry Models for Flame and Ignition Simulation," California Inst. of Technology, Rept. GALCITFM:2010.002, Pasadena, CA, 2010.

[25] Teng, H. H., Jiang, Z. L., and Ng, H. D., "Numerical Study on Unstable Surfaces of Oblique Detonations," Journal of Fluid Mechanics, Vol. 744, April 2014, pp. 111-128. https://doi.org/10.1017/jfm.2014.78

[26] Teng, H. H., and Jiang, Z. L., "On the Transition Pattern of the Oblique Detonation Structure," Journal of Fluid Mechanics, Vol. 713, Dec. 2012, pp. 659-669. https://doi.org/10.1017/jfm.2012.478

[27] Ziegler, J. L., Deiterding, R., Shepherd, J. E., and Pullin, D. I., "An Adaptive High-Order Hybrid Scheme for Compressive, Viscous Flows with Detailed Chemistry," Journal of Computational Physics, Vol. 230 , No. 20, 2011, pp. 7598-7630. https://doi.org/10.1016/i.jcp.2011.06.016

[28] Cai, X. D., Liang, J. H., Deiterding, R., and Lin, Z. Y., "Detonation Simulations in Supersonic Combustible Mixtures with Nonuniform Species," AIAA Journal, Vol. 54, No. 8, 2016, pp. 2449-2462. https://doi.org/10.2514/1.J054653

[29] Mazaheri, K., Mahmoudi, Y., and Radulescu, M. I., "Diffusion and Hydrodynamic Instabilities in Gaseous Detonations," Combustion and Flame, Vol. 159, No. 6, 2012, pp. 2138-2154. https://doi.org/10.1016/j.combustflame.2012.01.024

[30] Cai, X. D., Liang, J. H., and Deiterding, R., "Numerical Investigation on Detonation Control Using a Pulse Hot Jet in Supersonic Combustible Mixture," Combustion Science and Technology, Vol. 188, No. 10, 2016, pp. 1674-1690.

https://doi.org/10.1080/00102202.2016.1193499 


\section{Queries}

1. AU: Please check that the copyright (@) type is correct. Please note that the code will be added upon publication.

2. AU: Definitions of acronyms have been moved from the nomenclature into the text of the article, per AIAA style.

3. AU: Please add complete reference for [31] to the References list.

\section{Funding Information}

The following research funding sources have been associated with your manuscript:

- National Natural Science Foundation of China; Award no. 11702323;

Funder ID http://dx.doi.org/10.13039/501100001809

Funding sources listed here can be 1) research grants from outside agencies or organizations or 2) if an author is employed by a U.S. government agency that directly funded this research, this employer also may be listed here as a funding agency. Please confirm that this information is complete and correct for all authors. Edit the Funding Data/Acknowledgments section of your paper if you have changes to funding agency names or grant numbers. Use the funder's full and official name. 